\title{
Microscopic Stress and Strain Evolved in a Twinning-Induced Plasticity Fe-Mn-C Steel
}

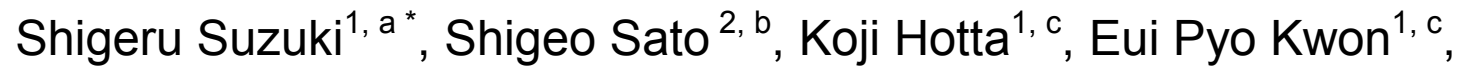 \\ Shun Fujieda ${ }^{1, d}$, Kozo Shinoda ${ }^{1, e}$, Kentaro Kajiwara ${ }^{3, f}$, Masugu Sato ${ }^{3, g}$ \\ ${ }^{1}$ Tohoku University, IMRAM, Katahira, Aoba-ku, Sendai 980-8577, Japan \\ 2 Tohoku University, IMR, Katahira, Aoba-ku, Sendai 980-9579, Japan \\ 3 Japan Synchrotron Radiation Research Institute, SPring-8, Hyogo 679-5198, Japan \\ $a^{a^{*}}$ ssuzuki@tagen.tohoku.ac.jp, bs.sato@imr.tohoku.ac.jp, ck.hotta@mail.tagen.tohoku.ac.jp, \\ cackep@mail.tagen.tohoku.ac.jp, ${ }^{d}$ fujieda@tagen.tohoku.ac.jp, ${ }^{\mathrm{e}}$ shinoda@tagen.tohoku.ac.jp, \\ fmsato@spring8.or.jp, ${ }^{\mathrm{f}} \mathrm{kajiwara@spring8.or.jp}$
}

Keywords: white x-ray diffraction, orientation, twining-induced plasticity, polycrystalline grains

\begin{abstract}
White X-ray diffraction with micro-beam synchrotron radiation was used to analyze microscopic stress evolved in coarse grains of a twinning-induced plasticity $\mathrm{Fe}-\mathrm{Mn}-\mathrm{C}$ steel under tensile loading. In addition, electron backscatter diffraction (EBSD) was used to determine the crystal orientation of grains in the polycrystalline $\mathrm{Fe}-\mathrm{Mn}-\mathrm{C}$ steel. Based on these orientation data, the stress and strain distribution in the microstructure of the steel under tensile loading was estimated using an FEM simulation where the elastic anisotropy or the crystal orientation dependence of the elasticity was taken into account. The FEM simulation showed that the strain distribution in the microstructure depends on the crystal orientation of each grain. The stress analysis by the white X-ray diffraction indicated that the direction of the maximum principal stresses at measured points in the steel under tensile loading are mostly oriented toward the tensile direction. This is qualitatively consistent with the results of by the FEM simulation, although absolute values of the principal stresses may contain the effect of heterogeneous plastic deformation on the stress distribution.
\end{abstract}

\section{Introduction}

It is well recognized that the mechanical properties of structural materials such as steel are influenced by the evolution of microscopic stress within their crystalline grains. Analytical methods for investigating such microscopic stress have been developed owing to advanced diffraction techniques. For instance, the microscopic stress and microstructure have been investigated using X-ray diffraction with micro-beam synchrotron radiation [1-4]. We have also applied a white X-ray diffraction method with micro-beam synchrotron radiation to analyze the microscopic stress evolved in grains of iron based shape memory alloys. The results showed characteristic microscopic stresses by tensile deformation at room temperature and annealing [5].

Austenitic steels containing a large amount of manganese, whose composition is similar to that of the iron based shape memory, exhibit good mechanical properties such as a high elongation and high ductility. The mechanical properties of these steels are sometimes associated with twinning-induced plasticity (TWIP) in which mechanical twins are formed [6]. The mechanical twins formed in TWIP steels are related with low stacking fault energy (SFE), which depends on the chemical composition of alloying elements such as manganese and carbon. In order to describe the SFE using the chemical composition of various alloying elements, thermodynamic studies of austenitic steels have been performed [7]. The effect of SFE on the microstructure, such as the crystallite size, during the deformation of TWIP steels has been studied by analyzing the line broadening in X-ray diffraction [8]. These results showed that the stacking fault probability is correlated with the crystallite sizes of different TWIP steels; however, the plastic deformation of the TWIP steels is influenced not only by 
twin formation but also by slip deformation due to dislocations. Fig. 1 illustrates the ideal twin formation in a face centered cubic (fcc) structure such as austenitic steels. The stress for the twin formation is dependent of the crystal orientation toward the stress direction. Typically, mechanical twins are relatively easily formed in a sample of [144] orientation toward the stress direction, where the Schmid factor is nearly the maximum value of 0.5 .

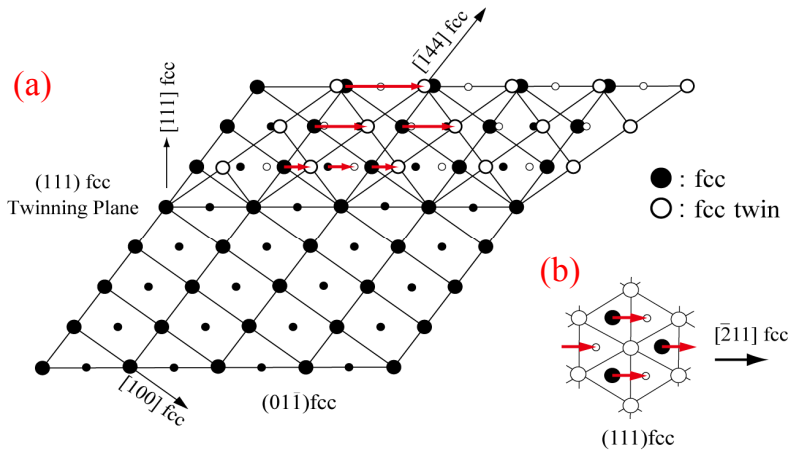

Fig. 1 Twin formation in a fcc structure induced by shear deformation as observed from views (a) and (b).

In order to understand the mechanism of twin formation and high ductility in TWIP steels, it is important to investigate the stress distribution in the microstructure in which both slip and twinning take place. Before the systematic measurements of microscopic stresses in differently strained samples are investigated, the stress distribution in a slightly deformed sample must be investigated. The objective of this study is therefore to analyze the stress distribution across different grains of a TWIP steel by white X-ray diffraction with micro-beam synchrotron radiation. The stress and strain distributions were also simulated by the finite element method (FEM). Since the elastic anisotropy of the fcc structure must be considered in an FEM simulation, electron backscatter diffraction (EBSD) was also used to determine the crystal orientation of the grains in the TWIP steel. The microscopic stress analyzed by the white X-ray diffraction is discussed in relation to the results estimated by the FEM simulation.

\section{Experimental and analysis procedures}

Sample Preparation. A button of an Fe-24\%Mn- $0.48 \% \mathrm{C}$ (mass $\%$ ) alloy was first prepared by arc melting in argon atmosphere [8]. The button of coarse grains was cut to prepare tensile samples with a gauge size of $3 \times 0.3 \times 1 \mathrm{~mm}^{3}$. After these tensile samples were annealed at $1223 \mathrm{~K}$, they were electrochemically polished carefully to obtain well-recrystallized samples. The samples had a large average grain size of $\sim 200 \mu \mathrm{m}$, which ensures the X-ray diffraction using micro-beam synchrotron for only one grain in the sample thickness direction.

Measurements. In situ X-ray diffraction experiments were performed at the BL28B2 of SPring-8 (Hyogo, Japan), where a high-energy white X-ray micro-beam was available. The X-ray energy was more than $50 \mathrm{keV}$ and the size was controlled to a $15 \mu \mathrm{m}$ diameter using a micro-hole. Laue patterns diffracted from a tensile sample with a low diffraction angle $(2 \theta)$ of $c a \cdot 0.1-20^{\circ}$ in the transmission Laue geometry were recorded during tensile testing using a flat panel detector [4]. For this measurement, small tensile samples mentioned above were used.

The diffraction data of the Laue patterns were recorded during tensile loading of $3.4 \%$ strain, and an amount of twins formed by this strain is negligible for the diffraction measurements. The tensile strain was measured from the displacement of marks on a tensile sample, and the applied load was simultaneously recorded using a load cell attached to the grip. X-ray energy spectra for several Laue reflection spots were measured using a solid-state detector [4,5]. The measured energy spectra were used to calculate the lattice spacing of the $(h k l)$ planes, that is $d^{h k l}$. Lattice strain in the $(h k l)$ planes, $\varepsilon^{h k l}$, was calculated from the change in $d^{h k l}$ with respect to the initial lattice spacing before deformation, that is $d_{0}$. In order to obtain grain images of a sample, all sequential diffraction 
experiments were processed by scanning the sample stage. As micro-beam white X-ray diffraction patterns are sensitive to the orientation of grains, the diffraction pattern changes significantly during stage scanning across grain boundaries of the sample. By comparing the change in the diffraction patterns numerically, it is possible to obtain grain images [3].

EBSD was utilized to analyze the crystal orientation of grains in the microstructure of the sample before deformation. The EBSD patterns were obtained using a Nordlys II EBSD detector mounted on a Hitachi SU-6600 scanning electron microscope. The crystal orientation obtained for each grain was used to estimate elastic constants of grains with an fcc structure. Using these elastic constants, an FEM simulation under plane stress conditions was carried out to estimate the stress and strain distribution in the polycrystalline sample. A software released by ANSYS Inc. was employed in the FEM simulation, in which an influence of the elastic anisotropy in the fcc structure was taken into account [8].

\section{Results and Discussion}

EBSD analysis and FEM simulation. Fig. 2(a) shows an inverse pole figure (IPF) map of the crystal orientation with respect to the tensile direction for five grains (A, B, C, D and E) in the present sample. The crystal orientation of these grains corresponds to the color denoted in the stereographic triangle shown in Fig.2 (b). The crystal orientations of these same five grains to the tensile direction in the microstructure are plotted in Fig. 3. Fig. 4 shows a contour plot of the Schmid factors for twin and slip deformations in the fcc structure. The orientation of the maximum Schmid factor for twinning differs from that for slip deformation, since the shear directions of twinning and slip deformation are [121] and [110], respectively [8]. This contour plot indicated that twin formation easily occurs in grains with an orientation near [101]-[111] toward the tensile direction, and that the slip deformation easily occurs in grains with other orientations [8].

(a)

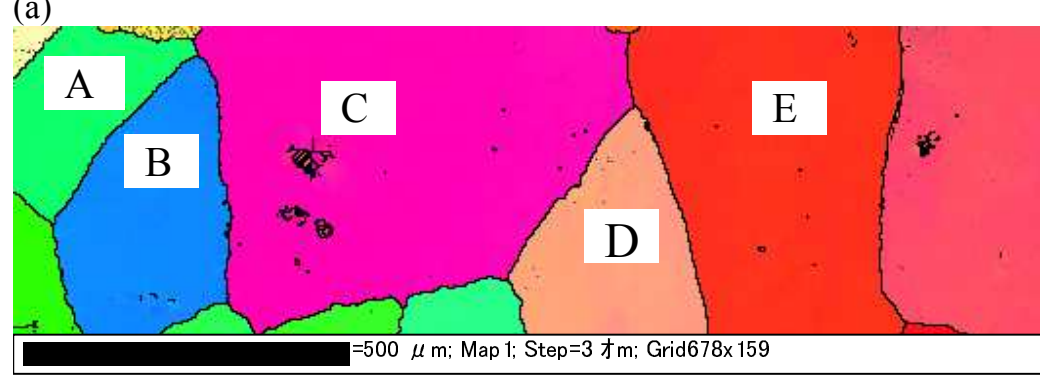

(b)

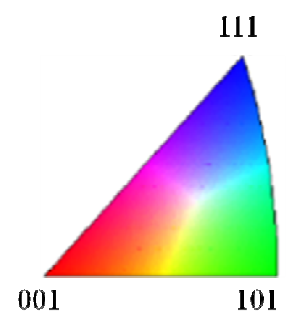

Fig. 2 (a) IPF map toward the tensile direction of the present sample and (b) a stereo triangle showing the crystal orientation.

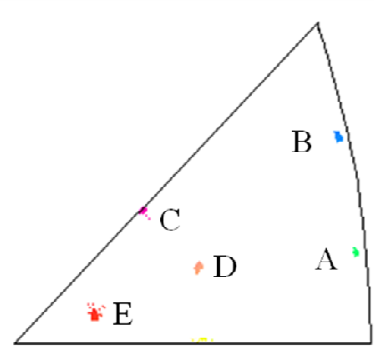

Fig. 3 The orientation of grains A, B, C $\mathrm{D}$ and $\mathrm{E}$ with respect to the tensile direction.

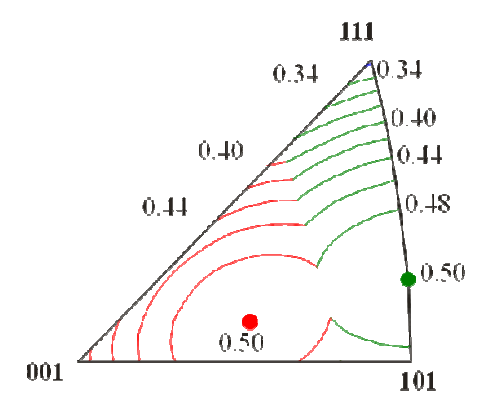

- Max. Schnid factor for twin - Mas. Schmid factor for slip

Fig. 4 Contour plot of Schmid factors for twin (green) and slip (red) deformations. 
In this study, the stress and strain distributions were simulated for the microstructure shown in Fig.2, in which the tensile direction of the sample was horizontal and the sample was deformed by $3.4 \%$. It was assumed that the crystal orientation dependence of elastic constants for each grain is same as that of a fcc-structured steel [5]. It was considered that the yield stress is $250 \mathrm{MPa}$ and the work hardening rate is $700 \mathrm{MPa}$ after yielding [8].

Fig. 5 (a) shows the direction of the maximum principal stress for each point in the area as shown in Fig. 2. The direction of the maximum principal stress is in agreement with the tensile loading direction, although the direction of the maximum principal stress may be influenced by local strain in a largely and heterogeneously strained sample. Fig. 5 (b) and (c) show contour maps of the maximum principal stress and the corresponding strain in the same area, respectively. The stress and strain levels in these contour maps are denoted as the colored scales in lower parts of the maps. The positions of grains A, B, C, D and E shown in Fig. 2 are denoted in Fig. 5. The stress distribution simulated here is relatively homogeneous in relation to the microstructure of the polycrystalline sample, although some stress concentration is observed close to grain boundaries. However, the strain induced by tensile loading is found to be dependent on the different grains. Typically, the strain is large in grain E while it is small in grain A, as shown in Fig. 5 (c). This phenomenon mainly arises from the crystallographic anisotropy of Young's modulus in the fcc structure. In other words, Young's modulus is low in [100] oriented grains, and it is high in [111] oriented grains. While the stress and strain distributions were estimated under simple assumptions by the FEM simulation, the realistic microscopic stress should be analyzed experimentally to understand the heterogeneity of deformation of the present sample.

(a)

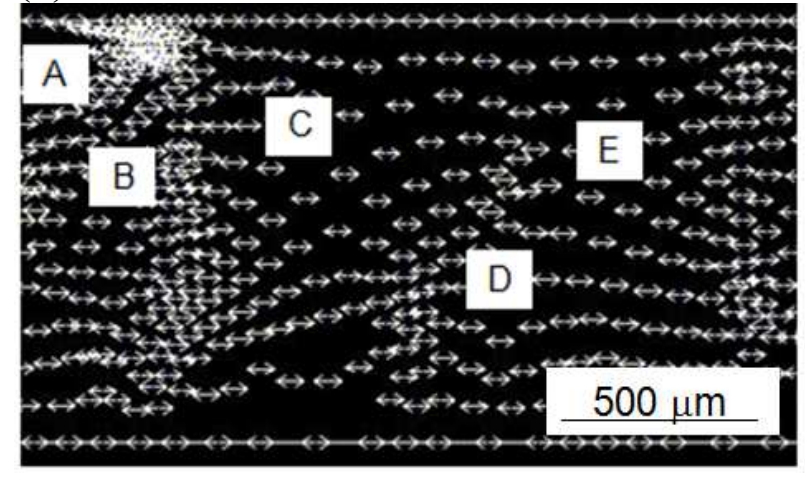

(b)

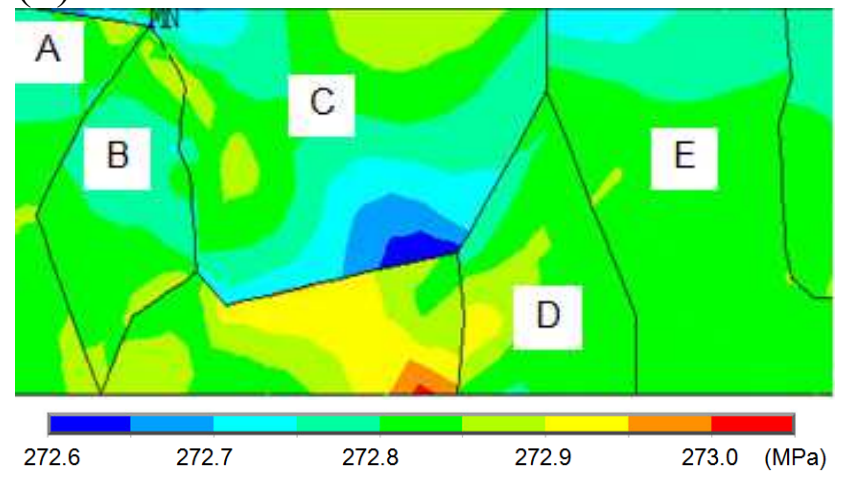

(c)

Fig. 5 (a) The direction of the maximum principle stress of each point in the microstructure, (b) the principal stress distribution toward the tensile direction and (c) the corresponding strain in the microstructure.

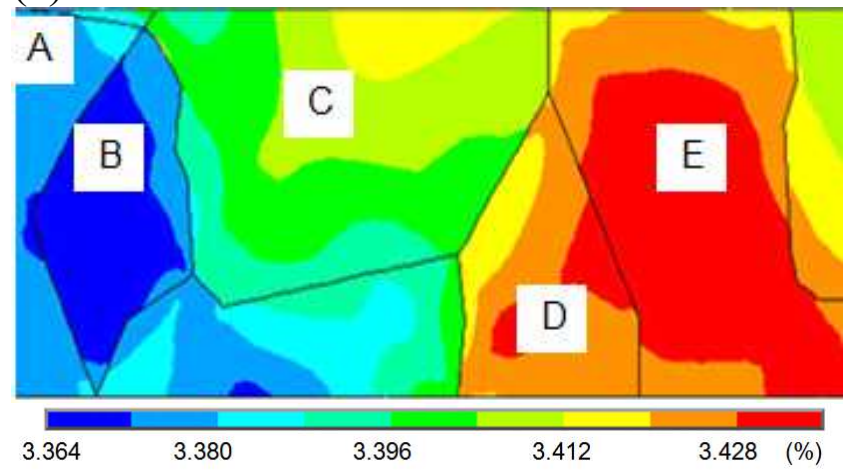

Microscopic stress analysis. The aforementioned results indicate that the crystal orientation of each grain to the tensile loading direction is an important factor in the microscopic stress and strain distribution in polycrystalline samples. Twin formation in this sample strained up to $3.4 \%$ by tensile loading was not clearly observed by EBSD analysis. However, it is possible to analyze the microscopic stress evolved in grains with different orientations under tensile loading using the X-ray 
diffraction with synchrotron radiation. Fig. 6 shows the experimentally measured maximum principal stresses in several grains a polycrystalline sample under about $40 \mathrm{~N}$ loading. The directions of the maximum principal stresses are indicated as arrows. The grain images of the unloaded and tensile-loaded sample were obtained though the analysis of a large number of Laue patterns. The image position in the tensile-loaded sample is slightly different from that of unloaded sample because of small deformation. The image contrast due to grain boundaries seem to be unclear, indicating that grain boundaries were not necessarily perpendicular to the sample surface. Despite this limitation, the measured area is almost same as the image observed by EBSD as shown Fig.2. The microscopic stress analysis indicates that the direction of the principal stresses at these points in the sample under loading is almost oriented to the tensile direction. This is fundamentally consistent with the result obtained of the FEM simulation. However, the maximum principal stress at a few points is not oriented to the tensile direction. This suggests that external stresses are released by slip deformation due to dislocations. In addition, it should be noted that the measured stress level is different from the simulated stress level shown in Fig.5(b). This difference is considered to result from several assumptions and parameters such as the initial lattice parameters used in the stress analysis and FEM simulation.

(a)

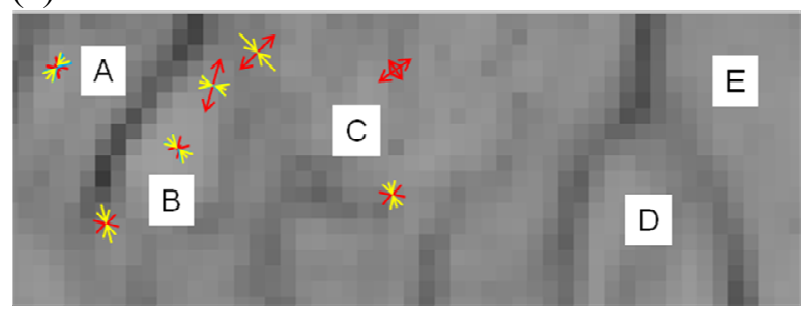

(b)

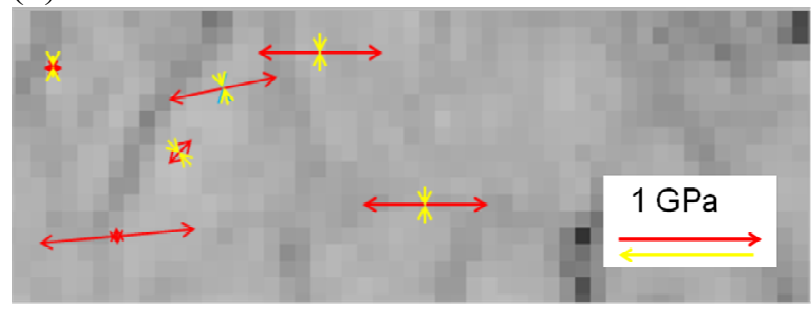

Fig. 6 The maximum principal stress at several points in the microstructure under (a) unloading and (b) tensile-loading conditions. Red and yellow color arrows denote tensile and compressive stresses, respectively.

Thus, the stress distribution estimated by the FEM simulation is fundamentally similar to the microscopic stress analyzed by micro-beam white X-ray diffraction, although there are some differences in these stress levels. These differences are also considered to arise from the heterogeneous plastic deformation due to dislocations. For instance, it is known that a large number of heterogeneous dislocations, which are called with geometrically necessary dislocations [9], are generated close to interfaces such as grain boundaries and twin boundaries. Such heterogeneous plastic deformation may influence measured stress level in stress analysis, since the overall average lattice spacing was analyzed in this study. The heterogeneous deformation may also induce the local stress concentration in grains, in which mechanical twins are initiated. Therefore, further microscopic stress analysis is now in progress for differently strained TWIP steels, in which mechanical twins are formed. It is expected that those results would provide information on some hints to understand the characteristic mechanical properties of TWIP steels.

\section{Summary}

The stress and strain distribution evolved in the microstructure of a polycrystalline TWIP Fe-Mn-C steel under tensile loading was estimated by the FEM simulation, in which the elastic anisotropy was considered. The FEM simulation indicated that the direction of the maximum principal stresses is oriented to the tensile direction, and characteristic strains are distributed in the microstructure. The micro-beam white X-ray diffraction was used for analyzing the maximum principal stresses evolved in grains of the TWIP steel under tensile loading. The results indicated that the direction of the 
maximum principal stresses in the steel during tensile loading is mostly oriented to the tensile direction, which is fundamentally similar to the results estimated by the FEM simulation. Although there are some differences of stress levels between simulation and experimental results, the differences may arise from heterogeneous microscopic plastic deformation.

Acknowledgements This study was supported by a Grant-in-Aid for Scientific research of the Japan Society for the Promotion of Science. The synchrotron radiation experiments were performed on the BL28B2 at SPring-8 with the approval of the Japan Synchrotron Radiation Research Institute.

\section{References}

[1] N. Tamura, H. A. Padmore and J. R. Patel, High spatial resolution stress measurements using synchrotron based scanning X-ray microdiffraction with white or monochromatic beam, Mater. Sci. Eng. A, 399 (2005) 92-98.

[2] F. Hofmann, X. Song, T.-S. Jun, B. Abbey, M. Peel, J. Daniels, V. Honkimäki and A. M. Korsunsky, High energy transmission micro-beam Laue synchrotron X-ray diffraction,, Mater. Lett., 64 (2010) 1302-1305.

[3] K. Kajiwara, M. Sato, T. Hashimoto, I. Hirosawa, T. Yamada, T. Terachi, T. Fukumura and K. Arioka, Development of visualization method of grain boundaries in stainless steel by using white X-ray micro-beam and image detector, Phys. Status Solidi A 206 (2009) 1838-1841.

[4] K. Kajiwara, M. Sato, T. Hashimoto, T. Yamada, T. Terachi, T. Fukumura and K. Arioka, Evaluation of internal stress in individual grains of cold-rolled stainless steel by energy dispersive X-ray diffraction,, ISIJ International, 53 (2013) 165-169.

[5] E.P. Kwon, S. Sato, S. Fujieda, K. Shinoda, K. Kajiwara, M. Sato, S. Suzuki, Microscopic residual stress evolution during deformation process of an Fe-Mn-Si-Cr shape memory alloy investigated using white X-ray microbeam diffraction, Mater. Sci. Eng. A, 570 (2013) 43-50.

[6] A. Dumay, J.-P. Chateau, S. Allain, S. Migot, O. Bouaziz, Influence of addition elements on the stacking-fault energy and mechanical properties of an austenitic Fe-Mn-C steel, Mater. Sci. Eng. A 483-484 (2008) 184-187.

[7] S. Allain, J. -P. Chateau, O. Bouaziz, S. Migot, N. Guelton, Correlations between the calculated stacking fault energy and the plasticity mechanisms in Fe-Mn-C alloys, 387-389 (2004) 158-162.

[8] S. Sato, E.P Kwon, M. Imafuku, K. Wagatsuma, S. Suzuki, Microstructural characterization of high-manganese austenitic steels with different stacking fault energies, Mater. Charact., 62 (2011) 781-788.

[9] A. J. Wilkinsonm T. B. Britton, Strains, planes, and EBSD in materials science, Materials Today, 15 (2012), 366-376. 